BMJ Open The association between alcohol

\title{
drinking and self-reported mental and physical functioning: a prospective cohort study among City of Helsinki employees
}

To cite: Salonsalmi A, Rahkonen 0, Lahelma E, et al. The association between alcohol drinking and selfreported mental and physical functioning: a prospective cohort study among City of Helsinki employees. BMJ Open 2017;7:e014368. doi:10.1136/ bmjopen-2016-014368

- Prepublication history and additional material is available. To view please visit the journal (http://dx.doi.org/ 10.1136/ bmjopen-2016-014368).

Received 22 September 2016 Revised 8 February 2017 Accepted 22 March 2017
CrossMark

${ }^{1}$ Department of Public Health, University of Helsinki, Helsinki, Finland

${ }^{2}$ Finnish Centre for Pensions, Helsinki, Finland

Correspondence to

Ms. Aino Salonsalmi;

aino.salonsalmi@helsinki.fi

\section{ABSTRACT}

Objectives Alcohol drinking is associated with ill health but less is known about its contribution to overall functioning. We aimed to examine whether alcohol drinking predicts self-reported mental and physical functioning 5-7 years later.

Design A prospective cohort study.

Setting Helsinki, Finland.

Participants 40-year-old to 60-year-old employees of the City of Helsinki (5301 women and 1230 men) who participated in a postal survey in 2000-2002 and a followup survey in 2007.

Primary and secondary outcome measures Mental and physical functioning measured by the Short Form 36 Health Survey.

Results Alcohol drinking was differently associated with mental and physical functioning. Heavy average drinking, binge drinking and problem drinking were all associated with subsequent poor mental functioning except for heavy average drinking among men, whereas only problem drinking was associated with poor physical functioning. Also, non-drinking was associated with poor physical functioning. Problem drinking was the drinking habit showing most widespread and strongest associations with health functioning. The associations between problem drinking and poor mental functioning and with poor physical functioning among women remained after adjusting for baseline mental functioning, sociodemographic factors, working conditions and other health behaviours.

Conclusions Alcohol drinking is associated especially with poor mental functioning. Problem drinking was the drinking habit strongest associated with poor health functioning. The results call for early recognition and prevention of alcohol problems in order to improve health functioning among employees.

\section{INTRODUCTION}

Alcohol drinking plays a significant role in the aetiology of many acute and chronic diseases, ${ }^{1}$ and it causes a major burden to population health accounting for $4 \%$ of disability adjusted life years worldwide. ${ }^{2}$
Strengths and limitations of this study

- The strength of the study was its prospective design which allowed us to consider previous health functioning. Alcohol drinking was measured by several variables and we were also able to take into account several covariates.

- The data were large and included both women and men.

- The study only included middle-aged municipal employees, and thus caution is needed when generalising the findings.

However, not only are the diseases important but also their effects on well-being and health functioning. Health functioning has increasingly been in the focus when examining patient groups and a similar approach has gradually spread to studies on general populations as well.

Previous studies on alcohol drinking and health functioning have found impaired health functioning among heavy drinkers measured by sickness absence ${ }^{34}$ and disability retirement ${ }^{56}$ for example. Less is known about how alcohol drinking contributes to self-evaluated general health and personal well-being. Previous studies have suggested impaired self-reported mental and physical functioning among patient groups. Furthermore, abstinence and treatment for alcohol abuse and dependence have been associated with improved functioning. ${ }^{78}$ Nevertheless, examining general populations is important as the heaviest burden of alcohol drinking falls on moderate drinkers, as they constitute a much larger group in number. ${ }^{910}$ For example, a Finnish study found that among men $64 \%$ and among women $93 \%$ of alcohol-related deaths occurred among the lower $90 \%$ of alcohol drinkers ${ }^{10}$ and a study 
from Norway found that the majority of acute alcohol problems were found among the lower $90 \%$ of alcohol drinkers. ${ }^{9}$

Studies on alcohol drinking and health functioning among general populations have been scarce. An Australian longitudinal study examined elderly women and found that non-drinkers had lower health functioning compared with moderate drinkers both in mental and physical functioning. ${ }^{11}$ Also, many cross-sectional studies have found poorer health functioning among non-drinkers compared with moderate drinkers. ${ }^{12-16}$ Results concerning heavy drinkers have been inconsistent: another Australian longitudinal study focusing on 45-year-old to 50-year-old women found that both non-drinkers and heavy drinkers had poorer health functioning compared with moderate drinkers. ${ }^{17}$ A Danish study examined both mental and physical functioning and found that heavy drinkers had the poorest mental functioning whereas non-drinkers had poorest physical functioning. ${ }^{18}$ However, a Finnish longitudinal study examining Finnish men in high socioeconomic positions found no differences in either mental or physical functioning between non-drinkers, moderate drinkers and heavy drinkers. ${ }^{19}$ In addition, some cross-sectional studies have found poorer health functioning among heavy drinkers, ${ }^{20}$ whereas some have suggested that heavy drinking is associated with better health functioning. ${ }^{121621}$

The studies on alcohol drinking and health functioning have mainly focused on the overall amount of alcohol consumption and seldom included measures such as binge drinking or problem drinking. There is increasingly evidence that in addition to the amount of alcohol consumed also drinking habits contribute to the effects of alcohol drinking. For example, binge drinking has been associated with mental health ${ }^{22}$ and mortality ${ }^{23}$ independently of the total amount of alcohol consumed.

This study examines the associations between alcohol drinking and subsequent mental and physical functioning among middle-aged municipal employees in a prospective study design. The study includes three variables on alcohol drinking, namely weekly average drinking, binge drinking and problem drinking. Women and men are studied separately and baseline health functioning, sociodemographic factors, working conditions and other health behaviours are taken into account.

\section{DATA AND METHODS}

\section{Study population}

This study is part of the Helsinki Health Study on middleaged employees of the City of Helsinki in Finland. The City of Helsinki is the largest employer in Finland and the jobs include a variety of both manual and non-manual job tasks such as secretaries, healthcare workers, teachers and garden workers. The baseline data were collected in three separate surveys in 2000, 2001 and 2002 by sending postal questionnaires to employees of the City of Helsinki in Finland who reached the age of 40, 45,
50,55 or 60 years during those years. ${ }^{24}$ A total of 8960 employees responded yielding a response rate of $67 \%$. A follow-up survey including essentially the same questions was conducted in 2007 with a response rate of $83 \%$ and 7332 respondents. The majority of the participants, $80 \%$, were women, which reflects the gender distribution of the employees in the Finnish municipal sector. Younger employees, men, those in lower socioeconomic position and those with previous sickness absence history were slightly underrepresented but non-response analyses suggest that major bias is unlikely. ${ }^{24} 25$

In this study, the baseline and follow-up data were combined. After exclusions due to being pregnant at baseline and missing data on health functioning or the covariates, this study included 5301 women and 1230 men. Due to item non-response on questions on drinking habits, the final analyses include slightly less participants. The final numbers are presented in table 1 .

The study protocol was approved by the ethics committees of the Department of Public Health and the University of Helsinki and the health authorities of the City of Helsinki.

\section{Measures on alcohol drinking}

Measures on alcohol drinking were derived from the baseline survey. From the questions concerning the average use of beer, cider, wine, other mild beverages and spirits, we calculated the weekly average use of alcohol defining 1 unit as $12 \mathrm{~g}$ of pure alcohol. The participants were divided into non-drinkers (those drinking 0 units per week), light drinkers ( $<4$ units per week), moderate drinkers (4-16 units per week) and those drinking $\geq 16$ units. For men, we also included a group drinking $\geq 24$ units. The cut-points of 16 and 24 units are used as limits for heavy drinking for women and men in Finland. ${ }^{26}$

Binge drinking was measured by a question asking how often the respondent drank $\geq 6$ units of alcohol on a single occasion. The group of binge drinkers included also those binge drinking less often than once a month. In addition, non-drinkers were distinguished from non-bingers, and thus the binge variable included three categories: non-drinkers, non-binge-drinkers and binge drinkers. The number of non-drinkers was slightly different from the weekly average drinking variable as we used the answer 'I do not use alcohol' to ask about the frequency of drinking instead of drinking 0 units per week as a definition to being a non-drinker.

Problem drinking was measured by the CAGE questionnaire ${ }^{2728}$ deriving from four questions: Have you ever thought about Cutting down your drinking? Have you ever felt Annoyed about criticism about your drinking? Have you ever felt Guilty because of your drinking? and Have you ever needed an Eye-opener? A positive answer to each question adds one point to a summary score ranging from 0 to 4 . For women, we used the conventional cut-off of 2 but for men we used the cut-point of 3 instead as has been done in previous Finnish studies. ${ }^{26}$ With cut-off 2 nearly $40 \%$ of men had been classified as problem 
Table 1 The distributions of drinking habits

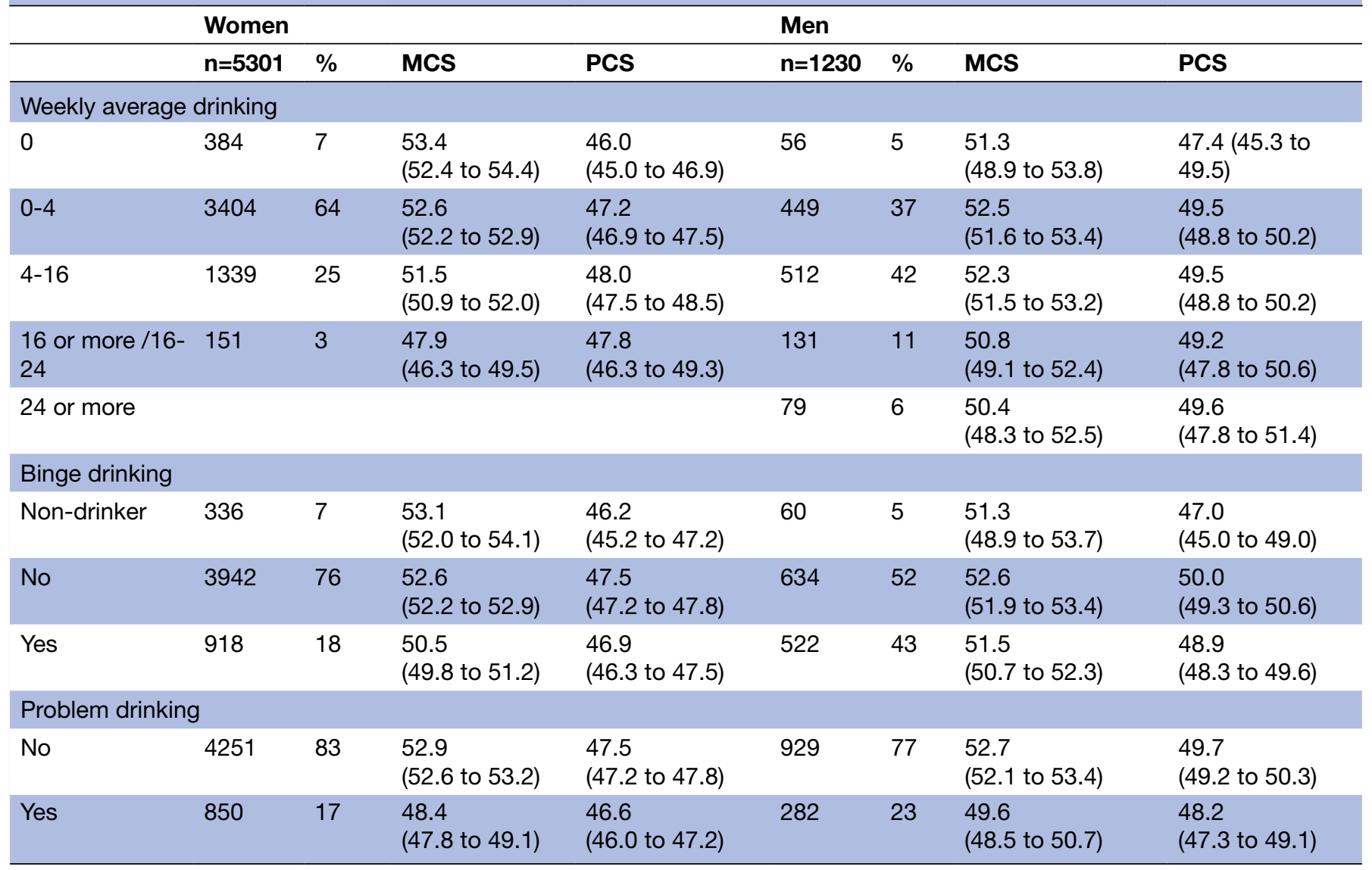

The age-adjusted mean scores and their $95 \%$ Cls for mental and physical component summaries MCS, mental component summary; PCS, physical component summary.

drinkers and we wanted to separate those with most serious drinking problems.

\section{Mental and physical functioning}

Mental (MCS) and physical (PCS) component summary scores of the Short Form 36 Health Survey were used as measures of mental and physical functioning in this study. ${ }^{29}$ MCS and PCS measure generic health and are also referred as health-related quality of life. The Short Form 36 Health Survey includes 36 questions on health and health-related functioning during the preceding 4 weeks. The eight subscales of the Short Form Health survey include physical functioning, role limitations due to physical problems, bodily pain, general health perceptions, mental health, role limitations due to emotional problems, social functioning and vitality. The subscales contribute positively or negatively to MCS and PCS. These scales are continuous, range from 0 to 100 and have been standardised to the US general population with a mean of 50 years and SD of 10 . Higher scores indicate better functioning than lower scores.

\section{Covariates}

The covariates were measured at baseline. Marital status was classified as single, married or cohabiting, and widowed or divorced. Socioeconomic position was categorised into managers and professionals, semiprofessionals, routine non-manual employees and manual employees. Mental and physical work load were both measured by single-item questions inquiring how mentally or physically demanding the respondent perceived her/ his work. Body mass index was divided into three groups: $<5$, between 25 and 30 and $>30 \mathrm{~kg} / \mathrm{m}^{2}$. Physical activity was measured by a 4-item questionnaire from which metabolic equivalent tasks were calculated. Those with fewer than 14 MET hours per week were classified as inactive and others as active. Smoking was divided into smokers and non-smokers. The MCS and the PCS at baseline were used as covariates and the scores were divided into gender-specific quartiles.

\section{Statistical methods}

The MCS and the PCS at follow-up were used as outcome variables and drinking habits at baseline as exposure variables. First, we calculated the age-adjusted mean scores for the MCS and the PCS and their 95\% CI by drinking habits. Second, we used logistic regression analysis to examine the associations between drinking habits and poor mental and physical functioning. In these analyses, the mental and physical component scores were dichotomised using the lowest gender-specific quartiles as 
cut-points for poor mental and physical functioning. We decided to dichotomise MCS and PCS scores as they were highly skewed and in addition our particular interest was in poor health functioning.

In the modelling, we first adjusted for age. Second, baseline MCS was added to the models examining mental functioning and baseline PCS to the models examining physical functioning. Third, we added other covariates to these models, first marital status, socioeconomic position and working conditions and then health behaviours. Women and men were studied separately due to differences in their average health functioning score levels and drinking habits. The results are presented as OR and their $95 \%$ CI. SAS V.9.3 was used in carrying the analysis.

\section{RESULTS}

The vast majority of participants were light or moderate drinkers drinking up to 16 units per week. $64 \%$ of women drank $<4$ units per week, whereas among men drinking 4-16 units per week was most common (table 1). Binge drinking and problem drinking were common especially among men. $18 \%$ of women were binge drinkers and $17 \%$ were problem drinkers. Among men the corresponding figures were $43 \%$ and $23 \%$. Among women heavy drinkers, binge drinkers and problem drinkers all had lower MCS scores compared with moderate drinkers, non-bingeing drinkers and non-problem drinkers. Among men problem drinkers had lower MCS and also PCS scores than non-problem drinkers. Concerning the PCS scores, both female and male non-drinkers had lowered scores than moderate drinkers.

Among women, heavy average drinking was associated poor mental functioning (OR 1.54, CI 1.08 to 2.19), whereas non-drinking $(0.73,0.56$ to 0.97$)$ and light drinking $(0.92,0.79$ to 1.08$)$ were associated with better mental functioning (table 2). Adjusting for mental functioning at baseline explained these associations. Binge drinking was associated with poor mental functioning $(1.49,1.27$ to 1.74$)$ and the association remained after adjusting for baseline mental functioning. Adjusting for marital status, socioeconomic position and working conditions had no effect but other health behaviours explained the association. The association between problem drinking and poor mental functioning was strong $(2.19,1.87$ to 2.56$)$ and remained after adjusting for baseline mental functioning. Other covariates had no effect on the association.

Non-drinking was associated with poor physical functioning compared both to moderate drinkers (1.61, 1.25 to 2.08$)$ and non-bingers $(1.31,1.02$ to 1.67$)$ among women. Also light drinking was associated with poor physical functioning compared with moderate drinkers (1.29, 1.11 to 1.51 ). The associations were however attenuated by baseline physical functioning. Problem drinking was associated with poor physical functioning even after all adjustments (1.22, 1.01 to 1.48$)$.
Among men, heavy weekly average drinkers tended to have poor mental functioning compared with moderate drinkers but the association did not reach statistical significance (table 3). Binge drinking was associated with poor mental functioning $(1.48,1.13$ to 1.95$)$ but the association was attenuated to statistically non-significant level after adjusting for baseline mental functioning. Non-drinkers had poor mental functioning compared with moderate drinkers and non-bingeing drinkers but this did not reach statistical significance. Problem drinking almost doubled the risk of poor mental functioning and the association remained even after all adjustments (1.50, 1.09 to 2.05).

In models adjusted for age only, problem drinking was associated with poor physical functioning (1.37, 1.02 to 1.84 ) but baseline physical functioning explained the association. After adjusting for baseline physical functioning also, binge drinking was associated with poor physical functioning but especially other health behaviours explained the association. Non-drinkers had poorer physical functioning compared with non-bingers (2.01, 1.15 to 3.51 ) but baseline physical functioning attenuated the association.

\section{DISCUSSION}

We examined the associations between alcohol drinking and subsequent self-reported mental and physical functioning. Alcohol drinking was differently associated with mental and physical functioning. Heavy weekly average drinking, binge drinking and problem drinking were all associated with subsequent poor mental functioning except for heavy average drinking among men. Concerning physical functioning, non-drinking was associated with poor functioning and regarding adverse drinking habits only problem drinking was associated with poor physical functioning. Problem drinking was the drinking habit strongly associated with health functioning as it was associated with poor mental functioning among both women and men and with poor physical functioning among women even after all adjustments.

Our results suggest that adverse drinking habits are associated especially with poor mental functioning and to a lesser extent with poor physical functioning. The association between alcohol drinking and poor mental health is well-established. Alcohol drinking has been associated with mental distress ${ }^{30} 31$ and depression ${ }^{21}$ for example. However, when examining the association between alcohol drinking and mental health, a question about their temporal order is often raised. There is evidence that both alcohol drinking predicts poor mental health and poor mental health predicts drinking. ${ }^{32}$ In our study, problem drinking and among women also binge drinking were associated with self-reported poor mental functioning even after adjusting for baseline mental functioning suggesting that alcohol drinking predicted poor mental functioning and not vice versa although the possibility of reverse causation cannot be excluded. 


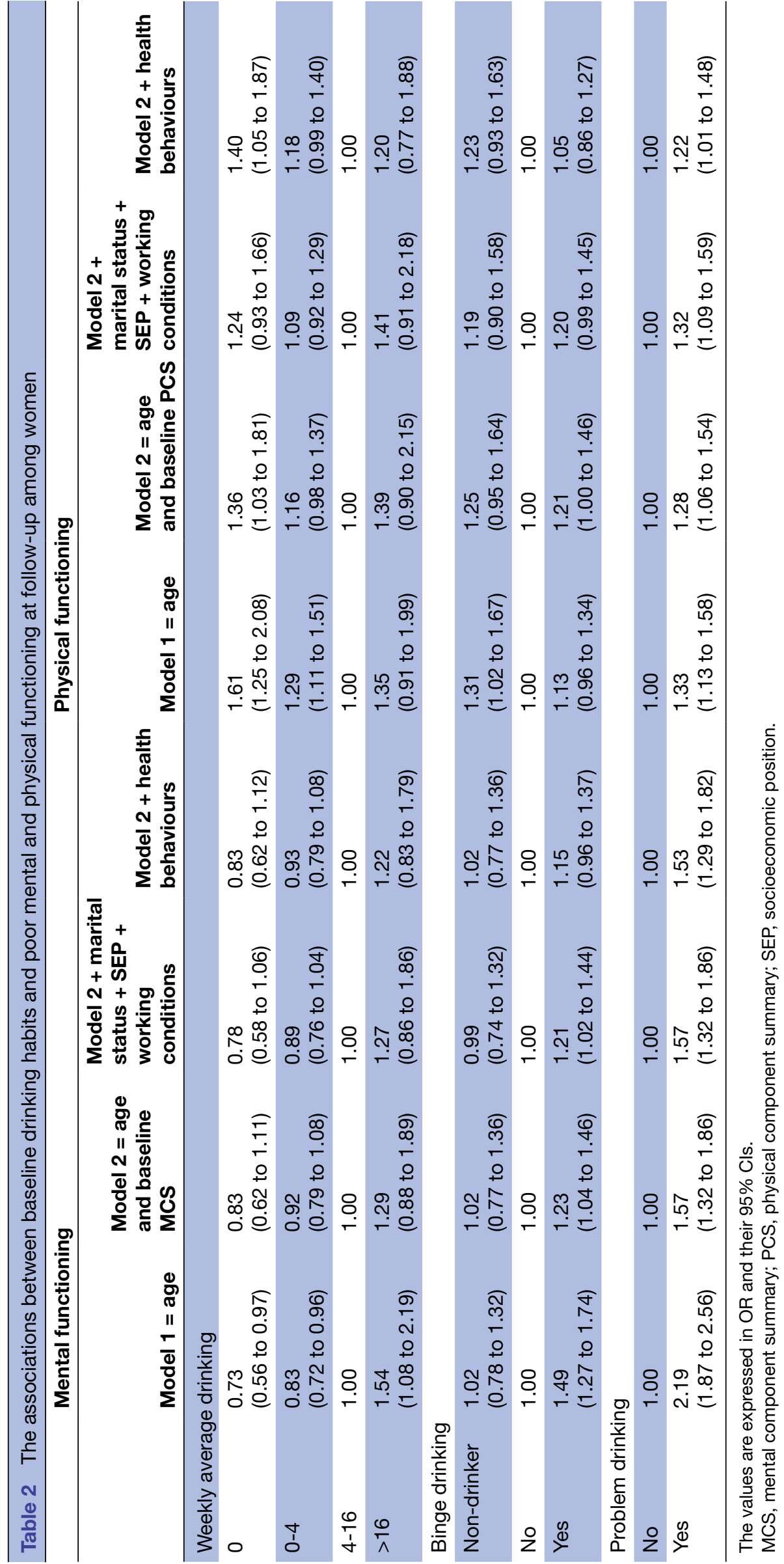




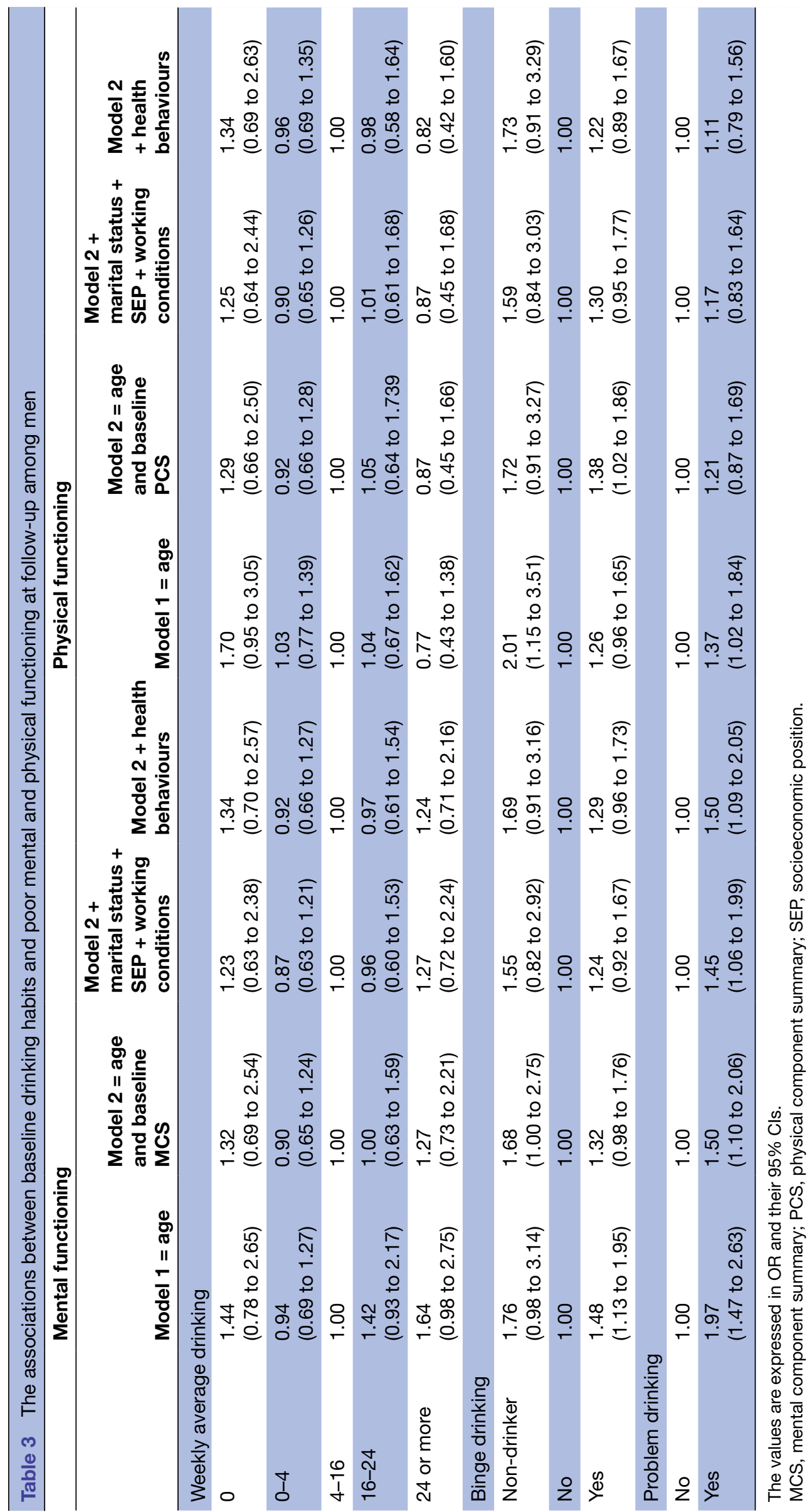


Although alcohol drinking is associated with many physical health problems such as gastrointestinal and neurological symptoms, in our study drinking habits had no widespread association with self-reported poor physical functioning. Only problem drinking and among men also binge drinking after adjusting for baseline physical functioning were associated with poor physical functioning. It might also be that poor mental functioning had an influence on the reporting of physical functioning also as poor among problem drinkers. In line with our study, a previous longitudinal study on Danish general population found that heavy drinking is associated with poor mental functioning but not with poor physical functioning, ${ }^{18}$ whereas a previous Finnish longitudinal study found no association between alcohol drinking and mental or physical functioning. ${ }^{19}$ Cross-sectional studies examining both mental and physical functioning have produced inconsistent results with either better mental $^{131521}$ or physical ${ }^{13151621}$ functioning or poorer mental ${ }^{20}$ functioning among drinkers.

Previous studies on alcohol drinking and health functioning have quite often found no associations between adverse drinking and poor health functioning ${ }^{13} 14$ or even better health functioning among heavy drinkers. ${ }^{12} 151621$ One reason behind this lack of association between alcohol drinking and poor health functioning might be that previous studies have often measured only the overall amount of alcohol used and not included other measures of drinking habits such as binge and problem drinking. In previous studies concerning other health outcomes such as mortality ${ }^{23}$ and mental disorders, ${ }^{22}$ binge drinking has been associated with poor health irrespective of the total amount of alcohol consumed. Concerning disability retirement, it has been found that heavy average drinking was not associated with all-cause disability retirement whereas problem drinking was. ${ }^{33}$ Our previous study, however, found that in addition to problem drinking also, heavy average drinking was associated with disability retirement. ${ }^{5}$ However, in this study, problem drinking was the measure most often associated with poor health functioning suggesting that instead of mere amount of alcohol consumed problem drinking matters most for poor health functioning.

Many previous studies have found that non-drinkers have poorer health functioning compared with moderate drinkers. Similar associations have been found with many other outcomes such as self-rated health, ${ }^{34}$ sickness absence, ${ }^{3536}$ coronary disease ${ }^{137}$ and mortality. ${ }^{37}$ It has been suggested that the group of non-drinkers includes previous problem drinkers as well as people abstaining due to health reasons and this explains the finding. Moderate drinkers might also share other health-promoting behaviours. Some previous studies were able to separate previous drinkers from non-drinkers, but also in these studies non-drinkers had poorer health functioning than moderate drinkers. ${ }^{14}{ }^{16}$ In our study, non-drinkers had poorer physical functioning compared with moderate drinkers and non-binge drinkers. The associations however mostly disappeared after adjusting for baseline physical functioning. It might thus be that non-drinkers are selected by poor health instead of moderate drinking being beneficial for health. Concerning mental functioning non-drinking women had even better functioning compared with moderate drinkers.

The strengths of our study include prospective study design and large study population of employees with relatively moderate drop-out. Drinking habits were measured by self-reports and the respondents might have underestimated their drinking. ${ }^{38}$ Although the absolute amounts consumed may not be exact, we believe that the results, however, portray relative differences between non-drinkers, moderate drinkers and heavy drinkers. Concerning binge drinking, the cut-point of 6 units may have been too low for men and underestimate the findings among them. Our study population consisted of ageing employees whose consumption was relatively moderate measured by units per week. Thus caution is needed when generalising the findings.

The study emphasises the importance of alcohol drinking on health functioning among ageing employees as associations were found even at a moderate consumption level. Only two previous studies have focused on employees. A Japanese cross-sectional study on male employees found no associations between heavy drinking and poor health functioning. ${ }^{14}$ This might have been because of good access to occupational health services that recognised and prevented heavy alcohol drinking. However, a cross-sectional study on Swedish female employees found that self-reported excessive drinking was associated with poor health functioning. ${ }^{20}$ The results call for early intervention when tackling poor health functioning due to alcohol drinking. We had the opportunity to adjust for baseline health functioning and this explained a number of the associations suggesting that differences between those with adverse drinking habits and others had developed already earlier. We adjusted also for marital status, socioeconomic position, working conditions and other health behaviours but they did not have marked effects on the associations.

Alcohol drinking was associated especially with poor mental functioning. However, the associations were partly explained by baseline mental functioning. Especially problem drinking was associated with poor mental functioning even after considering the baseline situation. Problem drinking was also associated with physical functioning. Early intervention is important in promoting health functioning among employees in general and among problem drinking employees in particular.

Contributors AS, OR, EL and ML contributed to the planning of the study and analysis and commented on the manuscript text. AS conducted the analyses and drafted the first version of the manuscript.

Funding The Finnish Foundation for Alcohol studies, the Yrjö Jahnsson Foundation and the Juho Vainio Foundation supported this research.

Competing interests None declared. 
Ethics approval By the ethics committees of the Department of Public Health and University of Helsinki and the health authorities of the City of Helsinki.

Provenance and peer review Not commissioned; externally peer reviewed.

Data sharing statement There are no additional data available.

Open Access This is an Open Access article distributed in accordance with the Creative Commons Attribution Non Commercial (CC BY-NC 4.0) license, which permits others to distribute, remix, adapt, build upon this work non-commercially, and license their derivative works on different terms, provided the original work is properly cited and the use is non-commercial. See: http://creativecommons.org/ licenses/by-nc/4.0/

(c) Article author(s) (or their employer(s) unless otherwise stated in the text of the article) 2017. All rights reserved. No commercial use is permitted unless otherwise expressly granted.

\section{REFERENCES}

1. Rehm J, Baliunas D, Borges GL, et al. The relation between different dimensions of alcohol consumption and burden of disease: an overview. Addiction 2010;105:817-43.

2. Lim SS, Vos T, Flaxman AD, et al. A comparative risk assessment of burden of disease and injury attributable to 67 risk factors and risk factor clusters in 21 regions, 1990-2010: a systematic analysis for the Global Burden of Disease Study 2010. Lancet 2012;380:2224-60.

3. Vahtera J, Poikolainen K, Kivimäki M, et al. Alcohol intake and sickness absence: a curvilinear relation. Am J Epidemiol 2002;156:969-76.

4. Salonsalmi A, Laaksonen M, Lahelma E, et al. Drinking habits and sickness absence: the contribution of working conditions. Scand $J$ Public Health 2009;37:846-54.

5. Salonsalmi A, Laaksonen M, Lahelma $\mathrm{E}$, et al. Drinking habits and disability retirement. Addiction 2012;107:2128-36.

6. Kaila-Kangas L, Kivekäs T, Laitinen J, et al. Abstinence and current or former alcohol use as predictors of disability retirement in Finland. Scand J Public Health 2015;43:373-80.

7. Donovan D, Mattson ME, Cisler RA, et al. Quality of life as an outcome measure in alcoholism treatment research. J Stud Alcohol Suppl 2005;15:119-39.

8. Ugochukwu C, Bagot KS, Delaloye S, et al. The importance of quality of life in patients with alcohol abuse and dependence. Harv Rev Psychiatry 2013;21:1-17.

9. Rossow I, Romelsjö A. The extent of the 'prevention paradox' in alcohol problems as a function of population drinking patterns. Addiction 2006;101:84-90.

10. Poikolainen K, Paljärvi T, Mäkelä P. Alcohol and the preventive paradox: serious harms and drinking patterns. Addiction 2007;102:571-8.

11. Byles J, Young A, Furuya $H$, et al. A drink to healthy aging: the association between older women's use of alcohol and their healthrelated quality of life. J Am Geriatr Soc 2006;54:1341-7.

12. Van Dijk AP, Toet J, Verdurmen JE. The relationship between healthrelated quality of life and two measures of alcohol consumption. $J$ Stud Alcohol 2004;65:241-9.

13. Green CA, Perrin NA, Polen MR. Gender differences in the relationships between multiple measures of alcohol consumption and physical and mental health. Alcohol Clin Exp Res 2004;28:754-64.

14. Saito I, Okamura T, Fukuhara S, et al. A cross-sectional study of alcohol drinking and health-related quality of life among male workers in Japan. J Occup Health 2005;47:496-503.

15. Stranges S, Notaro J, Freudenheim JL, et al. Alcohol drinking pattern and subjective health in a population-based study. Addiction 2006;101:1265-76.

16. Valencia-Martín JL, Galán I, Guallar-Castillón P, et al. Alcohol drinking patterns and health-related quality of life reported in the spanish adult population. Prev Med 2013;57:703-7.
17. Powers JR, Young AF. Longitudinal analysis of alcohol consumption and health of middle-aged women in Australia. Addiction 2008;103:424-32.

18. Pisinger $C$, Ladelund $S$, Glümer $C$, et al. Five years of lifestyle intervention improved self-reported mental and physical health in a general population: the Inter99 study. Prev Med 2009;49:424-8.

19. Strandberg A, Strandberg T, Salomaa V, et al. 29-y total mortality, and quality of life in men in old age. Am J Clin Nutr 2004;80:1366-71.

20. Bendtsen P, Hensing G, Alexanderson K. Self-perceived excessive alcohol consumption among employed women: association with health and psychosocial factors. Addict Behav 2003;28:777-83.

21. Chan AM, von Mühlen D, Kritz-Silverstein D, et al. Regular alcohol consumption is associated with increasing quality of life and mood in older men and women: the Rancho Bernardo Study. Maturitas 2009;62:294-300.

22. Paljärvi T, Koskenvuo $\mathrm{M}$, Poikolainen $\mathrm{K}$, et al. Binge drinking and depressive symptoms: a 5-year population-based cohort study. Addiction 2009;104:1168-78.

23. Laatikainen T, Manninen L, Poikolainen $\mathrm{K}$, et al. Increased mortality related to heavy alcohol intake pattern. J Epidemiol Community Health 2003;57:379-84.

24. Lahelma $E$, Aittomäki $A$, Laaksonen $M$, et al. Cohort profile: the Helsinki Health Study. Int J Epidemiol 2013;42:722-30.

25. Laaksonen M, Aittomäki A, Lallukka T, et al. Register-based study among employees showed small nonparticipation Bias in health surveys and check-ups. J Clin Epidemiol 2008;61:900-6.

26. Aalto M. Prevalence and brief intervention of heavy drinkers in primary health care. Tampere: Tampere University Press, 2001.

27. Bisson J, Nadeau L, Demers A. The validity of the CAGE scale to screen for heavy drinking and drinking problems in a general population survey. Addiction 1999;94:715-22.

28. McCusker MT, Basquille J, Khwaja M, et al. Hazardous and harmful drinking: a comparison of the AUDIT and CAGE screening questionnaires. QJM 2002;95:591-5.

29. Ware JE, Kosinski M, Keller SD. SF-36 physical and mental component summary measures: a user's Manual. Boston, MA: The Health Institute, New England Medical Center, 1994.

30. Saarni SI, Joutsenniemi K, Koskinen S, et al. Alcohol consumption, abstaining, health utility, and quality of life--a general population survey in Finland. Alcohol Alcohol 2008;43:376-86.

31. Mathiesen EF, Nome S, Eisemann M, et al. Drinking patterns, psychological distress and quality of life in a norwegian general population-based sample. Qual Life Res 2012;21:1527-36.

32. Swendsen JD, Merikangas KR. The comorbidity of depression and substance use disorders. Clin Psychol Rev 2000;20:173-89.

33. Skogen JC, Knudsen AK, Mykletun A, et al. Alcohol consumption, problem drinking, abstention and disability pension award. the NordTrøndelag Health Study (HUNT). Addiction 2012;107:98-108.

34. Valencia-Martín JL, Galán I, Rodríguez-Artalejo F, Alcohol R-A Alcohol and self-rated health in a mediterranean country: the role of average volume, drinking pattern, and alcohol dependence. Alcohol Clin Exp Res 2009;33:240-6.

35. Upmark M, Möller J, Romelsjö A. Longitudinal, population-based study of self reported alcohol habits, high levels of sickness absence, and disability pensions. J Epidemiol Community Health 1999;53:223-9.

36. Salonsalmi A, Laaksonen M, Lahelma E, et al. Drinking habits and sickness absence: the contribution of working conditions. Scand J Public Health 2009;37:846-54.

37. Makelä P, Paljärvi T, Poikolainen K. Heavy and nonheavy drinking occasions, all-cause and cardiovascular mortality and hospitalizations: a follow-up study in a population with a low consumption level. J Stud Alcohol 2005;66:722-8.

38. Boniface S, Shelton N. How is alcohol consumption affected if we account for under-reporting? A hypothetical scenario. Eur J Public Health 2013;23:1076-81. 